\title{
Composing the Layer of Knowledge of Digital Technology in Architecture
}

\author{
Mikhael Johanes ${ }^{1, *}$, Yandi Andri Yatmo ${ }^{1}$ \\ ${ }^{1}$ Department of Architecture, Universitas Indonesia, Indonesia
}

\begin{abstract}
The use of digital tools in architectural practice has been evolving significantly. In following such developments, architectural practice has been incorporating digital technology not only to meet the current demand but also to pursue the vast amount of possibilities ahead. However, the integration of digital technology in architectural knowledge has been reasonably operative that produces uncritical understanding, and it tends to put architects as a passive user of technology. This paper argues that there are layers of knowledge that nees to be acknowledged and nourished accordingly in embracing the use of computation tools yet avoiding the overly simplistic.understanding. It attempts to explore the methods of digital technology in archietctural design practices as well as dicussions that follow to create a critical evaluation of its roles and potentials. The review is conducted theoretically in which the use of digital in the design process is explored in such a way to reveal its importance in architectural design methods. The review also crosses beyond the disciplines of architecture to construct more comprehensive understanding that bridges the logic of digital technology and architecture. The resulted map of methods of the digital thus can be used to develop a framework for digital discourse that bridge the operative knowledge of technology to the more critical perspectives.
\end{abstract}

Keywords: digital architecture, information, design method, architectural education

\section{Introduction: From Representation to Information}

Architects commonly use analogue representation such as drawings and models as a medium to materialise their ideas. It requires skill to relate the visual presentations of the medium as well as the tactile of hand involved with the real experience of the building for which takes years to develop during the training of an architect (Scheer, 2014). The materiality of the building and ideas is believed to relate directly to the representation that used by the architects. Various stages of architectural production processes determined the mode of representation that depends on its intention of creation (Robbins and Cullinan, 1994). For instance, architects often use a series of vogue sketches in the early stages of the design process where the ideas are synthesised from different consideration. Meanwhile, the abstract yet clear conventional construction drawing is used in the final phase of design in which the precise delivery of information to construction stage is necessary. The practice of architecture thus shapes its internal system of representation that enables the communication and production of knowledge that is nourished in architectural education systems.

Digital technologies "abstractly schematize" the analogic quality of representation into bits of information, structured and processed into its medium
(Allen, 1997). The use of digital technology in architecture transforms its representational medium into an information system. This transformation inevitably changes the way architects work with their ideas in architecture. The traditional representational system enables architects to articulate their thoughts by cultivating the immediacy between hand and paper (Picon, 2003). On the other hand, the information of architecture within the digital medium enables particular operations in a specific way according to its mode of information and underlying system (Christenson, 2016) which both allow new possibility yet causes difficulties. This change is somewhat inevitable in the current architectural practice as well as our everyday life. The ubiquity of information enabled by Internet shapes our primary perception thus shifts our physical reality as well. The changing of the architectural working medium from meaning-seeking representation platform into performance-based information system perhaps become the only options that we have now in responding to such development.

This paper argued that the development of information based architecture profoundly challenges the notion of materiality in architecture. Picon (2003) argues that digital development redefines our relation to materiality by changing our perception and behaviour through its medium. The immediacy of human gesture in sketches and drawings which allows intuitive coupling

Corresponding author: mikhael.johanes@eng.ui.ac.id 
between ideas in its representation provide convenience medium for architects to work. In contrast, the structured and systematic nature of digital medium creates a thick layer of complexity that breaks the convenience. The following sections thereby attempt to break down these thick conceptual layers of digital medium in which the materiality of architecture is transformed into information to put it into different "reality".

\section{The materiality of Architectural Information}

Mastering the use of computers does not mean that we need to learn all program available in the market. In contrast, the ability to work with computers is measured by the capability to extend its functionality beyond its designated utilisation. We can attain this capacity if we can understand how the information is processed and manipulated in its digital medium. In other words, it is necessary to understand the materiality of the digital information analogous to building elements on the building construction. Computers create the new "perceptual entities" and objects that enable architects to manipulate different kind of transformation that is unavailable to traditional means of architectural representation (Picon, 2003). While the perception of architecture in the conventional representational medium often oscillates between concrete experience and abstract ideas of materiality, digital tools fundamentally alter this relation by creating a different realm of materiality.

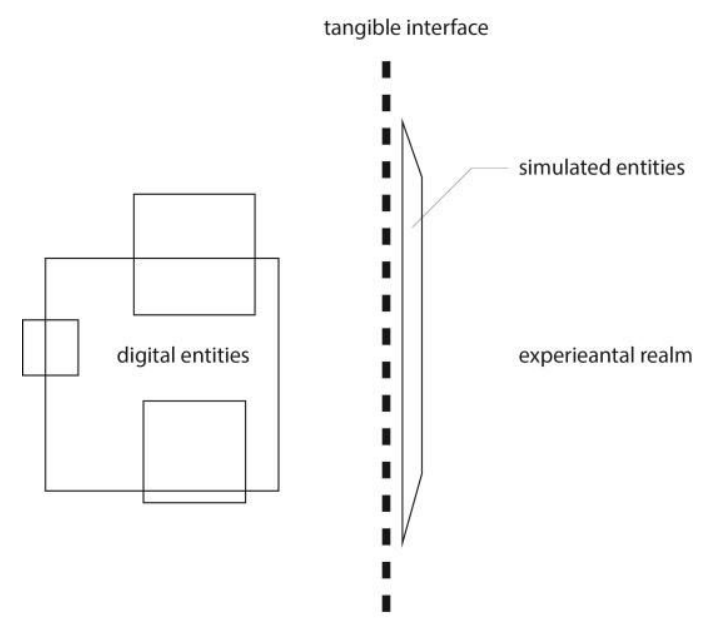

Fig. 1. Two Essential Elements of The Digital Medium.

We can conceive the digital medium such as computers according to its two essential elements: the surface demarcated by the interface on which people can interact with the medium and its interior that is the underlying system that works behind our vision (Figure 1). In its surface, the visible interface of digital medium creates a simulated experience in which its entities are perceived as reality, or even becomes the new reality itself (Scheer, 2014). The typical manifestation of this kind of digital surface is found in a smartphone, where the abstract information is experienced as reality using its intuitive touchscreen. The ever increasing development of virtual reality marks our fascination toward this simulated environment. This progression of highly simulated digital environment inevitably affects the practice of architecture as well as our lives in general. This ubiquity of simulated materiality thereby creates limited understanding because it mostly perceived as simulated experience and left its underlying abstract entities behind.

The interior of digital medium has less fascinating existence for people but perhaps holds more critical roles in understanding the digital materiality. The invisible, abstract mathematical entities (Emmer, 2005) inside the computer are the building blocks in digital architecture that are located within this interior as information. This information is somehow abstract, but its computability promises a wide range of opportunity if one can understand its operations. However, the current digital culture is seemingly flocking into the same direction in which a lot of things is done by similar blueprints with lack of inventiveness (Axel Killian in Burry, 2014). This condition reflects the shallow understanding of digital tools which only scratches the surface of digital without really digging deep by into its interior state. We argue that the knowledge of the digital should go beyond this surface, cultivating the space of abstract information within its medium that can sustain this development.

Christenson (2016) creates an analogy between grain in the physical objects to the materiality of digital information in architectural practice. He reveals that each mode of information has a different way of interpreting "reality" into digital environment thus affecting the kind of "force" that can be applied to it. Unlike the real physical objects that respond to natural forces such as gravity, weathering, and resistance in unity; the digital entities fragments and structures the reality into the various modes of information which can only be affected by specific operation enabled by its underlying systems. The more profound understanding of the way of information operation in digital architecture becomes crucial both to expose its limit and reveals its wide range of possibilities.

\section{The Operational Layer of Architectural Information}

The use of digital technologies is inevitably operative. However, the passive use of technologies puts its real operational capacity still intact, in which the nourished knowledge is more often only touching its surface of the interface. We propose that there are layers of operation by which the information of architecture potentially cultivated in an active manner, allows the tactical use of technologies instead of surrender to the given strategies (Figure 2). Those layers are the generation, translation, materialisation and simulation. Generation involves the operation of creation of digital entities which argued critical in the ideation of architectural possibility yet often limited by the existence of digital interface. Translation enables the fragmented nature of digital entities into the realm of conversations, to complement each other thus develops a richer architectural information system. Materialization and digitalization 
enable a specific mode of translation from and to digital realm into our physical realities. Those specific translations are argued to have important roles in bridging the two realms of architectural materiality. Simulation enables digital entities to reflects reality by performing it according to particular laws. While the generated data has significant value in anticipating the real performance of architecture, more critical evaluation of its law is arguably importance thus open architectural practice to a broader field.

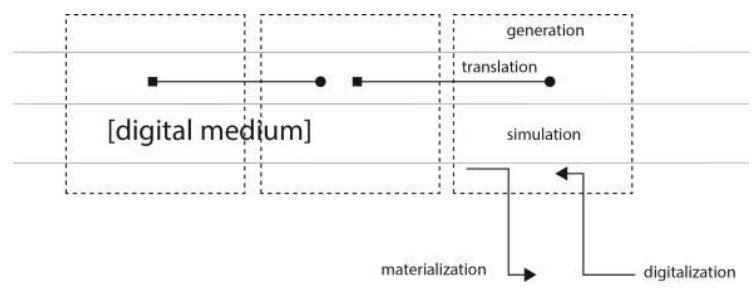

Fig. 2. Layers of Operation by The Information of Architecture.

\section{Generation}

Using digital medium requires the ability to generate desirable entities in which those objects convey particular information. There are three generation methods that dominate the current digital architectural process: directly creating using specific designated application, parametric systems and scripting (Dunn, 2012). Generating information using the assigned computer program is a pretty straightforward approach. In this method, the process is enabled but constrained by the interface of the program. For example, creating freeflowing continuous surface is more feasible using NURBS based modelling application rather that polygon based modelling application that is more appropriate to develop arbitrary surfaces from a collection of faces, edges and vertices (Corazza, 2012). This method requires the simulated environment to present the created abstract information directly to application's interface. Interfaces that both enable and limit the interactions between digital medium and its users define the very action that can be performed thus tend to hinder us for cultivating its latent possibilities.

Parametric systems enable architects to explore different possible outcomes from one abstract formal system by changing the finite value set of parameters. Parametric models are built from a set of components that embody specific operation, assembled as such to perform a somewhat complicated process and dealing with its abstract data structure (Woodbury, 2010). Parametric systems can extend the process of information generation beyond the designated interfaces by deliberately composing them. The tricky part of creating parametric system lies on the configurations of operations and the generated data to establish a desirable semantical relationship (Christenson, 2009). Fundamentally, creating a parametric system is a construction of a mathematical function that transforms a set of finite parameters into visible geometry. Mathematics thus holds an essential position in the establishment of such systems (Legendre, 2011) due to its very abstract nature behind the instrumentality of computation. Parametric systems bring mathematics into life by enabling operation that allows people to get results from its conceptual process in the form of geometry representation. However, people tend to avoid the difficulties in tackling this kind of abstracts problem and constraint itself using easy but limited operation in a well-designated application.

While the creation of architecture geometrical information using a specific application as well as parametric systems is performed through a welldesignated interface, scripting allows us to bypass the limitation and interface to perform the particular operation defined by specific syntactical computer language called codes (Burry, 2014). Script exploits the procedural process of computation to generate architectural information from its logical cores. It enables people to extend the operation of an application that is not defined in its interface yet possible. In other words, scripting opens a wide array of possibilities of action enabled by its medium rather than specific application. Understanding computer codes require the abstraction of procedures in which the process is composed which is not easy for everyone. The dichotomy between user and programmer creates a paradigmatic gulf that hinders the pollination between digital medium and architecture (Derix and Izaki, 2014). However, Kay (2012) argues that the digital language perhaps holds important roles in the future of learning analogous to literacy system that enables the forming of past civilizations. Once the difficulties of reading and writing of digital entities are surpassed, it amplifies our ability to cover different ground thus enable us to think differently.

\section{Translation and Interoperability}

Christenson (2016) describes translation as the act of interpreting information into another form such as interpreting a text as a visual image. In translation, the loss is inevitable, because each mode of data is designated as such according to its intended meaning and delivery purpose. Translation only allows particular information to be passed. In the case of conversion from text to an image, it neglects the textual data and maintains its graphical appearance. The loss also implies the translation is sometimes irreversible, which means we can never recover the missing information once it performed.

Each mode of information suggests a different operation according to its particular logic. Translation is not supposed to be seen as a form of loss. Instead, it enables various procedure that extends the meaning of initial information. Translation allows the abstract information to be processed "outside" its original form. In architectural processes in which the multiplicities of information are inevitable, translation enables transformative forces to be applied in the different situation thus provide a vibrant area of experimentation where new connections and associations are made 
(Christenson, 2016). Translation is argued as necessary in digital architecture to form a more diverse architectural design process.

Interoperability between systems in digital medium enables a kind of translation that has a slightly different aim from the former. Interoperability is defined as the capability of the various system to exchange its data (Jeong, Eastman, Sacks and Kaner, 2009) and prevent data loss as much as possible. While the translation between different modes of information aims to changes its method of interpretation and operation, interoperability seeks to create a shared understanding of separate systems to minimise the loss of information then it can be processed in the entirely different system. The more complex information in one system, the more laborious exchanges to be performed because every system has its way in structuring its information. Standardisation of such complex data is thus necessary to face those difficulties. For instance, the use of universal exchange format in BIM industry enables the different platform to work with each other (Jeong et al., 2009). However, total standardisation potentially will restrict the possibility of a different system to have various features which limit the possibility of extending the capability of the digital medium. The differences are necessary to maintain the possibility of diverse extensions. Both translation and interoperability bridge those gaps and thus allows a healthy diverse digital ecosystem to grow and work together.

\section{Materialisation and Digitalisation}

Digital fabrication fundamentally is a form of translation that transforms geometry information from the digital medium to physical materials through machinic operation controlled by computers (Ox-man and Oxman, 2014). Digital fabrication requires the ability to transform digital abstract information into process-based information to be executed by specific machines. This stage involves the understanding of available material process such as cutting, subtractive, additive and formation fabrication (Kolarevic, 2003) as well as the strategies that available for specific materials (Beorkrem, 2013). The capability to differentiate the process of materialisation enables building construction industry to shift from mass production that is based on standardisation into mass customisation from which differentiated building elements can be produced economically. Digital fabrication in building industry extends the possibility of construction thus giving architects more space in realising their ideas.

Digital fabrication also enables architects to quickly materialise her ideas during the process using rapid prototyping (Oxman and Oxman, 2014). It is a form of translation between abstract digital entities that enables iteration between the abstract materiality of the digital and physical materiality within design process which can bridge the gap between these two domains. The bridging between digital and physical reality allows architects to reconfigure its architectural information systems according to the feedback from physical realisation thus giving the digital-enabled process for having a "reality check" during the process. Digital fabrication thereby makes architecture as both spatial and material practice, practically blurring the boundary between design and construction.

On the other end of translations between digital and physical, digitalization denotes the reverse vector of translation which enables realities to be denoted as information (Allen, 1997). The most of loss is tend to happen in this kind of translation in which idealisation and structuration are inevitable. However, the ability of the digital medium to manage a large sum of information enables it to be a better medium of working rather than writing and drawing. While writing frees us from the necessity of memorizing and enable us to think otherwise (Kay, 2012), digitalization extends this prosthetic capability even forward. What is more, digitalization extends our capability of thinking through computation.

\section{Simulation and Performativity}

Computation enables the digital medium to perform digital simulations. Simulation fundamentally is a replication of reality that provides the possibility to study real-world phenomenon without direct intervention to its real context (Groat and Wang, 2013). Digital simulation holds an instrumental value that conventional medium cannot offer. The importance of simulation lies in its ability to generate information and forming iterative process between design and analysis. Simulation enables architects to anticipate building performance (Scheer, 2014) before being built into reality. High-performance architecture becomes possible due to the available computation technology in optimising different aspects of the building that sometimes have competing values which is harder to solve using conventional means of calculation.

There is no universal simulation yet and perhaps there never will be. Different simulation is needed to analyse various aspects of performance and requires a different set of information. For example, simulating structural integrity of the building requires information on the structural system that is not available in the 3D modelling application that only gives geometrical information. Building Information Modeling (BIM) system was thus invented to overcome this limitation by fundamentally providing better information management system and enable interoperability between different systems. BIM systems create "mirrored" reality of a building by forming a structured information that covers almost every aspects of the building including spatial, material, structural, operational, cost, schedules and even life-cycle factors over long periods of time (Groat and Wang, 2013). The development of digital simulation has come to the point that almost every performative aspects can be managed, evaluated and finally anticipated.

However, there is a limit. No matter how sophisticated and complex the simulation created in replicating real-world phenomenon, it is only a mode of analysis that should not be confused with the reality 
itself. The knowledge that can be gathered is highly operative from which the only observable effects is already anticipated by the "law" that underlies behind the simulation (Scheer, 2014). While the "law" aim to explain and predict a particular real phenomenon, simulation is created to perform according to this law. The way to expand the understanding the of reality through simulation should begin from its underlying theoretical basis. The multidiscipline effort is important in which different disciplines can work together to develop collective understanding in performing a simulation. Simulation can be seen as a door to open dialogue outside the discipline of architecture where the assessment and evaluation of performance requires a fair amount of theoretical understanding. Simulation can ground architecture to its reality only if it is constantly evaluated and criticised. Otherwise, the detachment of architecture from the "reality" is perhaps inevitable.

\section{The Layers of Digital Knowledge: Beyond Rationale of Vision}

The development of digital has come to an era when digitalisation of architectural practice is inevitable. However, the current development mostly puts architectural materiality as a simulated experience of information using its interface. The information based architecture requires further understanding of how the actual logic of the digital works in the medium. Digital entities embody different materiality (Picon, 2003) in which architectural ideas can be generated. Generating architecture in a digital medium both enable and constraint architects in using its interface. Bypassing the constraint of the interface thus becomes crucial in opening a wide range of possibility that lies in the digital interior system. Derix and Izaki (2014) suggest that mathematical thinking and computer programming is necessary to create intuitive coupling between digital media and its user. Intuitiveness in using digital media does not come from a seamless interface between user and media, but from familiarising oneself to the logic of how information is interpreted and operated digitally.

The transformation of architectural communication from representation into information enables procedural mode of presentation of architecture. Parametric system and algorithmic architecture show that design can be presented as a set of procedures containing various values and rules. The resulted architectural forms thus are only consequences from such processes. Reviewing such architecture thereby cannot be judged solely based on its final result. Instead one has to evaluate the very logic of values and rules that operate behind. It is not impossible if the future of architectural design publications will contain sets of procedures showing the logic behind rather than a set of drawings that solely represent its formal consequences. This transition is perhaps the best bet for architecture to escape from the rationale of vision that already dominates the architectural practice from the beginning of its development (Eisenman, 1992).

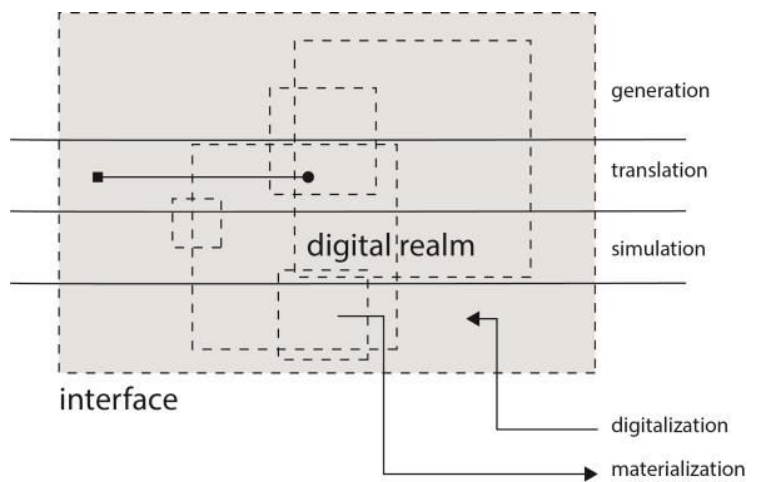

experiental realm

Fig. 3. Whole Layers of Digital Knowledge of Architecture.

The emergence of the digital process also enables architecture to reconsider its relation to materiality. The development of digital is often seen as a movement away from architectural materiality (Picon, 2003). It is true that the virtualization of architecture in the digital medium allows the material reality to escape from the grasp of our perception, but the dialogue of the digital and physical in digital medium goes beyond our perceptual experience. The fabrication process of digital lessens the gap of digital and physical where the material aspects inevitably should be taken into account. On the other hand, simulation puts the materiality of architecture in the medium of the digital domain in its abstract form based on particular theory or law. The theorization of materials enables a wide range of hypothesis to be tested before being realized into built forms. However, simulations and their underlying principles must be continuously checked to its real-world context empirically. Both simulations and fabrication oscillate the materiality of architecture within the realm of digital and physical, creating entanglement between both domains and forming the iterative architectural material practice.

Figure 3 shows the whole layers of digital knowledge of architecture. It can be conceived in two form which perhaps holds an essential role in the development of the architectural education curricula: the layers of the medium and the layers of methods. The layers of the medium denote the interface and interior in which the abstract entities inside should be understood, allow us to work in surpass the interface and work more tactically by understand its operating language. Computer language and its abstract foundational knowledge such as mathematics are arguably held important roles in digital practice as notation and convention also form the language of architectural representation. The layers of method denote the operational layers in which the entities of architectural information are generated and translated from and to the digital medium. Although those methods of operation can be performed in every layer of the digital medium from interface level to its deeper interior level, the deeper the layer of the medium used, the more possibilities can be founded thus provide a richer area of cultivation. On the other hand, these 
layers of methods also hold important roles in bridging the fundamental knowledge of digital medium with the more practical architectural design processes.

\section{Concluding Remark: Missing Information}

The transformation of architectural representation into information, however, has a limit in itself. The abstraction into digital realm forces architecture to be translated into entities and sets ontological equalities between them in which the status of relations between entities must be clarified (Loo, 2013). This translation implies that there will always be missing information in the digital architecture. The very abstracts nature of mathematical and algorithmic creates a stubborn reality of quantities has been limited itself in grasping the bodily feeling of spatiotemporal experiences (Parisi, 2013). Although digital transformation enables the materiality of architecture to shifts to new realm thus allowing it to evolve as such, the immaterial aspects of architectural materiality are yet still missing and perhaps can never be incorporated in the digital medium.

\section{References}

Allen, S. (1997). Object to Field. In M. Toy (Ed.), Architecture After Geometry (Vol. 67). Academy Press.

Beorkrem, C. (2013). Material strategies in digital fabrication. New York: Routledge, Taylor \& Francis Group.

Christenson, M. (2009). Testing the Relevance of Parameterization to Architectural Epistemology. Architectural Science Review, 52(2), 135. https://doi.org/10.3763/asre.2009.0022

Christenson, M. (2016). Beginning design technology. New York: Routledge, Taylor \& Francis Group.

Corazza, S. (2012). Modelling Techniques-Differences between NURBS and Polygon. Retrieved October 3, 2017 , from https:/seraphinacorazza.wordpress.com/2012/12/28/ modeling-techniques-differences-between-nurbs-andpolygon-modelling-new-york-city-rooftop-3darchitecture-project-research/

Derix, C., \& Izaki, A. (2014). New Curricula: Syntonic Systems. Architectural Design, 84(5), 122-129.

Eisenman, P. (2015). Architecture After the Age of Printing (1992). In M. Carpo (Ed.), The Digital Turn in Architecture 1992-2012 (pp. 15-27). Hoboken, NJ, USA: John Wiley \& Sons, Inc. https://doi.org/10.1002/9781118795811.ch1

Emmer, M. (2005). Mathland: The Role of Mathematics in Virtual Architecture. Nexus Network Journal, 7(2), 73-88. https://doi.org/10.1007/s00004-005-0023-1

Groat, L. N., \& Wang, D. (2013). Architectural research methods (2. ed. [updated and expanded]). Hoboken, NJ: Wiley.
Jeong, Y.-S., Eastman, C. M., Sacks, R., \& Kaner, I. (2009). Benchmark tests for BIM data exchanges of precast concrete. Automation in Construction, 18(4), 469-484.

https://doi.org/10.1016/j.autcon.2008.11.001

Kay, A. (2012). The Future of Reading Depends on the Future of Learning Difficult-to-Learn Things. In B. Junge, C. Zwick, \& Kunsthochschule BerlinWeißensee (Eds.), The digital turn: design in the era of interactive technologies. Zürich: Park books.

Kolarevic, B. (Ed.). (2003). Architecture in the digital age: design and manufacturing. New York, NY: Spon Press.

Legendre, G. L. (Ed.). (2011). Mathematics of space. London: Wiley.

Loo, S. (2013). Abstract Care. In H. Frichot \& S. Loo (Eds.), Deleuze and architecture (pp. 245-261). Edinburgh University Press.

Parisi, L. (2013). Contagious architecture: computation, aesthetics, and space. Cambridge, Massachusetts 1 London, England: The MIT Press.

Picon, A. (2003). Architecture and the virtual: towards a new materiality? Retrieved from https://e-pub.uniweimar.de/opus4/files/1236/picon.pdf

Robbins, E., \& Cullinan, E. (1994). Why architects draw. MIT press.

Scheer, D. R. (2014). The death of drawing: architecture in the age of simulation. London; New York: Routledge.

Woodbury, R. (2010). Elements of parametric design. London; New York, NY: Routledge. 